To Appear in the Astrophysical Journal Letters

\title{
The FIRST Radio-Loud Broad Absorption Line QSO and Evidence for a Hidden Population of Quasars
}

\author{
Robert H. Becker \\ University of California at Davis \\ and \\ Institute for Geophysics and Planetary Physics \\ Lawrence Livermore National Laboratory \\ bob@igpp.llnl.gov \\ Michael D. Gregg \\ Institute for Geophysics and Planetary Physics \\ Lawrence Livermore National Laboratory \\ gregg@igpp.llnl.gov \\ Isobel M. Hook \\ European Southern Observatory \\ imh@eso.org \\ Richard G. McMahon \\ Institute of Astronomy, Cambridge \\ rgm@ast.cam.ac.uk \\ Richard L. White \\ Space Telescope Science Institute \\ rlw@stsci.edu \\ David J. Helfand \\ Columbia Astrophysics Laboratory \\ djh@astro.columbia.edu
}

\begin{abstract}
We have discovered two low-ionization broad absorption line quasars in programs to obtain optical spectra for radio-selected quasar candidates from the VLA FIRST Survey (Becker, White, \& Helfand 1995). Both belong to the extremely rare class of BAL QSOs that exhibit narrow absorption lines from
\end{abstract}


metastable excited levels of Fe II and Fe III. Until now, there was just a single object in this class, 0059-2735 (Hazard et al. 1987). In addition, one of our new objects is the first known radio-loud $B A L Q S O$. The properties of these three unusual objects suggest a trend of increasing radio luminosity with the amount of absorption to the quasar, and are perhaps transition objects between radio-loud and radio-quiet quasars.

The two new objects are from a radio-selected sample comprising less than 200 quasars; one is heavily attenuated at optical wavelengths in the observed frame. These objects would be easily overlooked by most optical QSO searches; their abundance in the radio sample suggests that they may be representatives of a largely undetected component of the quasar population, perhaps as numerous as ordinary low-ionization BAL QSOs which constitute 1-2\% of all QSOs.

Subject headings: quasars: radio selected - quasars - galaxies: spectrophotometry

\section{Introduction}

Radio-loud quasars and broad absorption line (BAL) quasars (QSOs) each constitute $\sim 10 \%$ of the total quasar population (Foltz et al. 1990; Weymann et al. 1991) but have always appeared to be mutually exclusive classes (Stocke et al. 1992). There is little agreement about what determines quasar radio luminosities or what the relationship is between broad absorption lines and radio power. The BAL QSOs can be further subdivided into high and low-ionization objects (Hi-BALs and Lo-BALs). The Lo-BALs make up only $10 \%$ of the total BAL QSO population (Weymann et al. 1991) and are usually recognized by the presence of broad absorption from Si II, Mg II, and Al III. In the Lo-BAL population, there has for a decade been only one object, 0059-2735, which also shows narrow absorption lines from metastable excited states of Fe II and Fe III (Hazard et al. 1987). We report here the discovery of two more "iron Lo-BALs".

We have undertaken two programs to identify candidate quasars drawn from the NRAO円 Very Large Array (VLA) FIRST' Survey (Becker, White, \& Helfand 1995; White

\footnotetext{
${ }^{1}$ The National Radio Astronomy Observatory is operated by Associated Universities, Inc., under cooperative agreement with the National Science Foundation

${ }^{2}$ The FIRST Survey World Wide Web homepage is http://sundog.stsci.edu
} 
et al. 1997). Both programs select optical samples for spectroscopy by matching FIRST radio sources to stellar counterparts within 1".2 from the APM POSS I catalog (McMahon \& Irwin 1992). The FIRST Bright Quasar Survey (FBQS, Gregg et al. 1996) is developing a new, complete sample of radio-selected quasars brighter than 17.5 magnitude on the E plates and with $O-E<2.0^{3}$. The second program is searching specifically for high redshift quasars among very red optical counterparts with $O-E>1.0$ with the additional constraint that the sources also have flat radio spectra between 6 and $20 \mathrm{~cm}$ (Hook et al. 1996). The FIRST Survey quasar search programs are unique in that they go to much fainter radio levels than previous radio-selected QSO surveys: the $1 \mathrm{mJy}$ flux limit picks up radio-quiet objects out to $z \approx 2$. Roughly half of the objects in the FBQS pilot sample are, in fact, radio-quiet (Gregg et al. 1996).

For the past two years we have been taking $5 \AA$ resolution spectra of these quasar candidates using the Lick $3 \mathrm{~m}$ Shane telescope, the Kitt Peak National Observatoryf $2.1 \mathrm{~m}$ and $4 \mathrm{~m}$ telescopes, and the La Palma 2.5m Isaac Newton telescope. To date, 136 new quasars with $\mathrm{R} \lesssim 17.5$ have been found in the FBQS and $\sim 30$ additional quasars have been found in the high redshift program.

Among the new QSOs in the FIRST Survey samples, there are 4 BAL QSOs. Two are of the common Hi-BAL variety, but the other two, one from each program, have very unusual spectra, placing them in the iron Lo-BAL class with the heretofor unique object 0059-2735 (Hazard et al. 1987). Additionally, one of these two is the first known radio-loud BAL QSO.

\section{Observed Properties of $0840+3633$ and $1556+3517$}

\subsection{Optical Spectra and Photometry}

Optical spectra obtained with the Lick Observatory 3m telescope for FIRST J084044.5+363328 (0840+3633) and FIRST J155633.8+351758 (1556+3517) are displayed in Figure 1, shifted to the rest frame, along with the spectrum of 0059-2735 (from Weymann et al. 1991). Unlike most quasar spectra, which are characterized by strong, broad emission

\footnotetext{
${ }^{3}$ The POSS I O and E bands roughly correspond to the more familiar Cousins B and R. The APM magnitudes have an accuracy of $\sim 0.4$ magnitudes, though the colors are probably more accurate (McMahon \& Irwin 1992).

${ }^{4}$ Kitt Peak National Observatory, NOAO, is operated by the Association of Universities for Research in Astronomy, Inc. (AURA), under cooperative agreement with the National Science Foundation
} 
lines, these BAL QSO spectra are completely dominated by their absorption features; emission lines are relatively weak or absent. In Figure 1, the distinguishing absorption features are indicated; an asterisk denotes a metastable iron feature. In the spectrum of $0840+3633$, the unabsorbed remnant of the Mg II $2800 \AA$ emission line yields a redshift of 1.22. Its spectral features are very similar to those of 0059-2735, albeit with deeper absorption troughs, and it is unmistakably a more extreme example of this class of BAL QSO. The spectrum of $1556+3517$ has no prominent emission lines, but the absorption features are so similar to $0840+3633$ and $0059-2735$ that there is again no doubt that it is the same kind of object with even higher absorption. Not only are the broad absorption lines nearly saturated, but the continuum is heavily absorbed. The absorption features indicate a redshift of 1.48. In the two new BALs, the broad lines of Al II, Al III, and Mg II have nearly complete absorption in their cores. The absorption due to metastable excited lines of Fe II and Fe III (Hazard et al. 1987) is also deeper in the two new objects than in 0059-2735.

For $0840+3633$, the APM POSS I magnitudes are $\mathrm{O}=17.3$ and $\mathrm{E}=15.9$, while for $1556+3517, \mathrm{O}=21.2$ and $\mathrm{E}=18.7$. Both objects are redder than typical intermediate redshift quasars, for which $O-E \approx 0.5$ (FBQS). For 0059-2735, Hazard et al. (1987) estimate $\mathrm{R}=17.1$ and Hewett et al. (LBQS, 1995) list $B_{J}=18.13$. We adopt $\mathrm{H}_{\mathrm{o}}=50$ $\mathrm{km} \mathrm{s}^{-1} \mathrm{Mpc}^{-1}, \mathrm{q}_{\mathrm{o}}=0.1$, and an optical spectral index $\alpha_{\mathrm{opt}}=-1.0$ to compute the absolute R-band magnitudes. The pertinent data for the three iron Lo-BALs are listed in Table 1.

\subsection{Radio Properties}

Both new BAL QSOs are point sources in the FIRST catalog. The $1.4 \mathrm{GHz}$ flux densities for $0840+3633$ and $1556+3517$ are 1.3 and $30 \mathrm{mJy}$, respectively. The Greenbank $5 \mathrm{GHz}$ catalog (Becker, White, \& Edwards 1991) lists a flux density of $27 \mathrm{mJy}$ for $1556+3517$, giving a spectral index of -0.1 , assuming no variability. Stocke et al. (1992) provide an upper limit of $0.36 \mathrm{mJy}$ for the $5 \mathrm{GHz}$ flux density of 0059-2735. The flat radio spectrum for $1556+3517$ could be indicative of relativistic beaming, making a calculation of radio luminosity problematic, but we have computed $\mathrm{L}_{1400}$ for all three sources assuming isotropic radiation (Table 1) and adopting a spectral index of -0.1 .

We have computed the ratio of $5 \mathrm{GHz}$ radio to $2500 \AA$ optical flux, $\log (\mathrm{R})$, using equations 1-3 of Stocke et al. (1992). No K-corrections have been applied. The BAL $0059-2735$ is radio-quiet with $\log (\mathrm{R}) \approx 0,0840+3633$ is "radio-moderate" with $\log (\mathrm{R})=0.4$, $1556+3517$ has $\log (\mathrm{R})>3$. The usual dividing line between radio-loud and radio-quiet is $\log (\mathrm{R}) \approx 1$ (e.g. Stocke et al.), so, even with the uncertainties in the optical photometry, 
$1556+3517$ is the first known radio-loud $B A L Q S O$.

Could the conclusion that $1556+3517$ is radio-loud be a consequence of its large optical absorption? Its absolute B magnitude is 3.4 magnitudes fainter than 0059-2735 and $0840+3633$; if this difference is attributed entirely to attenuation, then $\log (\mathrm{R})$ drops to $\sim 1.8$, still a radio-loud object. Additionally, it has $\mathrm{L}_{1400}=10^{33.5} \mathrm{ergs} \mathrm{s}^{-1} \mathrm{~Hz}^{-1}$, well above the usual divide between radio-loud and radio-quiet of $10^{32.5}$ based on radio power alone (Schneider et al. 1992; Stocke et al. 1992).

\section{Discussion}

\subsection{Why Does FIRST Find Iron Lo-BALs?}

Francis, Hooper, and Impey (1993) have shown that BAL QSOs tend to be found closer to the radio-loud/radio-quiet divide than typical optically selected QSOs. They estimated that BAL QSOs are overabundant by a factor of 10 in the "radio-moderate" $(0.2<\log (R)<1.0)$ population compared to the radio-quiet population, and one of the brightest radio-moderates in their sample is a Lo-BAL, 1235+1807B. The low radio flux limit of the FIRST Survey leaves it sensitive to radio-quiet and radio-moderate objects with $z \lesssim 2$, so the presence of ordinary Lo-BALs in the FIRST quasar samples would not be surprising. Out of 170 newly identified quasars in the FIRST Survey searches, only two Lo-BALs have been found so far and both have highly unusual spectra placing them in the rare iron Lo-BAL class, defined by 0059-2735 (Hazard et al. 1987). In the current census of $\sim 8600$ quasars of Véron-Cetty \& Véron (1996), 0059-2735 is the only other such recognized object. The FIRST Survey is apparently more effective at finding these unusual quasars than previous surveys.

The rarity of iron Lo-BALs up until now can to some extent be attributed to the absence of UV excess or strong emission lines in their optical spectra as well as their relatively low radio flux densities. Even multicolor high- $z$ quasar surveys (e.g., Warren, Hewett, and Osmer 1994) have not detected this class of quasar. Although Hazard et al. (1987) point out that the appearance of 0059-2735 in their discovery objective prism data drew attention because of its strong Al II and Al III absorption, objects as heavily attenuated as 1556+3517 probably masquerade effectively as $\mathrm{M}$ stars in such surveys. The FBQS has a relatively weak color selection, including objects as red as $O-E=2$, while the high redshift survey intentionally targets even redder stellar objects, and neither rely

on objective prism data, so objects like $0840+3633$ and $1556+3517$ are not selected against in the optical. 
The spectra of the three iron Lo-BALs (Figure 1) suggest a correlation between column density of low-ionization clouds and radio emission. Based on the Fe II absorption lines and continuum attenuation, 0059-2735 has the lowest column density, followed by $0840+3633$, and then $1556+3517$; ranking by radio luminosity yields the same order. The severe attenuation of the continuum shape of $1556+3517$ also suggests the presence of large amounts of dust. Although derived from a very small sample, this correlation suggests that high column densities in these BAL QSOs are associated with stronger radio emission. The preponderance of known BAL QSOs have much lower column densities of low-ionization clouds, consistent with the relatively low radio power of all BAL QSOs discovered to date. If the correlation between extinction in the optical and radio luminosity is correct, then as the iron Lo-BALs become harder to detect in the optical, they become more prominent in the radio and will naturally be more plentiful in radio-selected samples. Based purely on these empirical results, we propose that most BAL QSOs are radio-quiet and will not show up in a radio-selected sample, while the iron Lo-BALs are a special subpopulation which occupy the transition region between radio quiet and radio loud objects. The discovery of two iron Lo-BALs out of $<200$ quasars suggests that they make up $\sim 1-2 \%$ of the FIRST quasars, comparable to the rate of Lo-BALs in the general quasar population as currently understood.

A possible additional high-redshift $(\mathrm{z}=2.33)$ member of the iron Lo-BALs is Hawaii 167, analyzed by Cowie et al. (1994) and Egami et al. (1996). The spectrum of Hawaii 167 (Figure 2 of Cowie et al.) does show marked similarities with 0059-2735 but lacks strong Mg II 2800 emission and does not have particularly broad absorption troughs. The discovery of the two new iron Lo-BAL objects eases the transition between 0059-2735 and Hawaii 167; 0840+3517 is intermediate while 1556+3517 has even less Mg II emission and a more attenuated continuum than Hawaii 167, though its absorption features appear broader. If our suggestion of a link between radio power and column density of absorbing material is correct, then Hawaii 167 should have a radio luminosity between that of $1556+3517$ and $0840+3633$. Hawaii 167 has not been detected in the $20 \mathrm{~cm}$ VLA NVSS Survey (Condon et al. 1996), implying an upper limit of $\sim 2.5 \mathrm{mJy}$ and $\log \left(\mathrm{L}_{1400}\right)<32.8$, leaving room for it to fit the trend.

Hawaii 167 is quite faint in the optical, $B=23.0$ (Cowie et al.). It was found in a complete spectroscopic survey of IR-selected objects in a region covering only $77 \operatorname{arcmin}^{2}$. Cowie et al. (1994) argue that finding such an object in a small area survey indicates that these objects may be quite common, albeit at faint optical magnitudes, consistent with the results of the FIRST quasar samples. 


\subsection{Dusty QSO Interpretation}

Voit, Weymann, \& Korista (1993) have proposed a model for Lo-BALs in which a nascent QSO is embedded in a region with a high rate of massive star formation, enshrouded with dust. In their scenario, these objects then evolve into more ordinary Hi-BALs with time, as their dust shrouds dissipate. If indeed $1556+3517$ were to evolve into a more ordinary BAL, its radio luminosity would have to diminish. Egami et al. (1996) extend this model to Hawaii 167 and 0059-2735, explaining the iron Lo-BALs as a combination of heavily reddened QSO + starburst. They point out that such objects would likely be missed by optical surveys because the high dust content suppresses the rest frame UV (observed optical) and because these objects lack strong emission lines. They further note that if the starburst activity has not begun, these objects will be "completely dark" in the optical, implying that there may be a large, undetected population of these hybrid starburst/quasar objects.

This model helps to explain the FIRST Survey sensitivity to iron Lo-BALs: our radio-selected samples are at least partly immune to the optical selection effects that may cause these objects to be overlooked. If so, the FIRST Survey is now picking up the brightest, and possibly lowest redshift, tip of the iceberg. These objects have not yet acquired strong emission lines, but have evolved to the point where they can be seen through the surrounding dust. Additional arguments for populations of undetected, dusty quasars have been put forth by Low et al. (1989), Sprayberry \& Foltz (1992), and Webster et al. (1995). Just how numerous the iron Lo-BALs are will be determined as the FIRST QSO surveys progress and IR surveys become more common.

\section{Conclusions}

We have discovered two low-ionization BAL QSOs with strong absorption from metastable excited states of Fe II and III ("iron Lo-BALs"). There has been just a single member of this class until now, 0059-2735, with Hawaii 167 another likely example. One of the new objects, $1556+3517$, is the first known radio-loud BAL QSO. The three iron Lo-BALs suggest a correlation between radio luminosity and absorption. We suggest that the iron Lo-BALs are transition objects evolving from radio-loud to radio-quiet BAL systems as the QSO emerges from the enshrouding material. The two new FIRST Survey objects and Hawaii 167 were all discovered in limited surveys at wavelengths longer than optical, suggesting that these objects escape detection in most optical surveys and may be common, perhaps equal in population to the radio-quiet Lo-BALs. 
Whether considered extreme or transition, the iron Lo-BALs may hold clues to several aspects of the quasar phenomenon, starburst activity in the early Universe, and the relation of both to galaxy formation. Egami et al. (1996) emphasize that the heavy obscuration of the central QSO allows the surrounding galaxy starlight to be detected, opening a window on QSO galaxy hosts at higher redshift. If this is accurate, then $1556+3517$ should be even more galaxy-like in the IR than Hawaii 167 because of its higher extinction of the QSO source. It is possible that these objects are a "missing link" between galaxies and quasars.

We acknowledge Jules Halpern for first recognizing the nature of $0840+3633$ and for additional helpful discussions. We thank Paul Hewett for the spectrum of 0059-2735 and Michael Brotherton for many useful comments and suggestions. We acknowledge support from the NRAO, the NSF (grants AST-94-19906 and AST-94-21178), the IGPP/LLNL (DOE contract W-7405-ENG-48), the STScI, the National Geographic Society (grant NGS No. 5393-094), NATO (grant CRG 950765), and Sun Microsystems. This paper is Contribution Number 620 of the Columbia Astrophysics Laboratory. 


\section{REFERENCES}

Becker, R.H., White, R.L., \& Edwards, A.L. 1991, ApJS, 75, 1

Becker, R.H., White, R.L., \& Helfand, D.J. 1995, ApJ, 450, 559

Condon, J.J., Cotton, W.D., Greisen, E.W., Yin, Q.F., Perley, R.A., Taylor, G.B., \& Broderick, J.J., in preparation

Cowie, L.L., Songaila, A., Hu, E.M., Egami, E., Huang, J.-S., Pickles, A.J, Ridgway, S.E., Wainscoat, R.J., \& Weymann, R.J. 1994, ApJ, 432, L83

Egami, E., Iwamuro, F., Maihara, T., Oya, S., \& Cowie, L.L. 1996, preprint, astro-ph 9604135

Foltz, C., Chaffee, F., Hewett, P., Weymann, R., Anderson, S., \& MacAlpine, G. 1989, AJ, 98, 1959

Francis, P.J., Hooper, E.J., \& Impey, C.D. 1993, AJ, 106, 417

Gregg, M.D., Becker, R.H., White, R.L., Helfand, D.J., McMahon, R.G., \& Hook, I.M. 1996, (FBQS), AJ, 112, 407

Hazard, C., McMahon, R.G., Webb, J.K., \& Morton, D.C. 1987, ApJ, 323, 263

Hewett, P.C., Foltz, C.B., \& Chaffee, F.H. 1995, AJ, 109, 1498

Hook, I.M., McMahon, R.G., Irwin, M.J., \& Hazard, C. 1996, MNRAS, in press

Low, F.J., Cutri, R.M., Kleinman, S.G., \& Huchra, J.P. 1989, ApJ, 340, L1

McMahon, R.G., \& Irwin, M.J. 1992, in Digitized Optical Sky Surveys, eds. H.T. MacGillivray and E.B. Thomson, Kluwer, p. 417

Schneider, Donald P., van Gorkom, J.H., Schmidt, M., \& Gunn, J.E. 1992, AJ, 103, 1451

Sprayberry, D., \& Foltz, C.B. 1992, ApJ, 390, 39

Stocke, J.T., Morris, S.L., Weymann, J.T., \& Foltz, C.B. 1992, ApJ, 396, 487

Véron-Cetty, M.-P. \& Véron, P. 1996, European Southern Observatory Scientific Report No. 10, A Catalog of Quasars and Active Nuclei, (Seventh Edition), in press

Voit, G.M., Weymann, R.J., \& Korista, K.T. 1993, ApJ, 95, 109

Warren, S.J., Hewett, P.C., \& Osmer, P.S. 1994, ApJ, 421, 412

Weymann, R.J., Morris, S.L., Foltz, C.B., \& Hewett, P.C. 1991, ApJ, 373, 23

White, R.L., Becker, R.H., Helfand, D.J., \& Gregg, M.D. 1997, ApJ, in press 
Table 1. BAL Quasar Vital Statistics ${ }^{a}$

\begin{tabular}{|c|c|c|c|c|c|c|c|c|c|c|}
\hline ID & $\mathrm{RA}_{(\mathrm{J} 2}$ & D0) & $\mathrm{z}$ & B & $\mathrm{R}$ & $\mathrm{M}_{\mathrm{R}}$ & $\begin{array}{l}\mathrm{S}_{1400}{ }^{\mathrm{b}} \\
(\mathrm{mJy})\end{array}$ & $\begin{array}{l}\mathrm{S}_{5000} \\
(\mathrm{mJy})\end{array}$ & $\begin{array}{c}\log \left(\mathrm{L}_{1400}\right) \\
\left(\operatorname{ergs~s}^{-1} \mathrm{~Hz}^{-1}\right)\end{array}$ & $\log \left(\mathrm{R}^{*}\right)$ \\
\hline $0059-2735$ & 010217.1 & -271951 & 1.62 & 18.1 & 17.1 & -28.9 & .. & $<0.36^{\mathrm{c}}$ & $<31.71^{\mathrm{e}}$ & $<0.04$ \\
\hline $0840+3633$ & 084044.5 & +363328 & 1.22 & 17.3 & 15.9 & -29.3 & 1.6 & $\ldots$ & 32.03 & 0.37 \\
\hline $1556+3517$ & 155633.8 & +351758 & 1.48 & 21.2 & 18.7 & -27.0 & 30.6 & $27.0^{\mathrm{d}}$ & 33.50 & 3.18 \\
\hline
\end{tabular}

${ }^{\mathrm{a}}$ for $\mathrm{H}_{\mathrm{o}}=50, \mathrm{q}_{\mathrm{o}}=0.1, \alpha_{\text {rad }}=-0.1, \alpha_{\text {opt }}=-1.0$

${ }^{b}$ FIRST Survey, Becker et al. 1995

${ }^{\text {c}}$ Stocke et al. 1992

dGB 5GHz Survey, Becker et al. 1991

${ }^{\mathrm{e}}$ Estimated from $\mathrm{S}_{5000}$ upper limit 


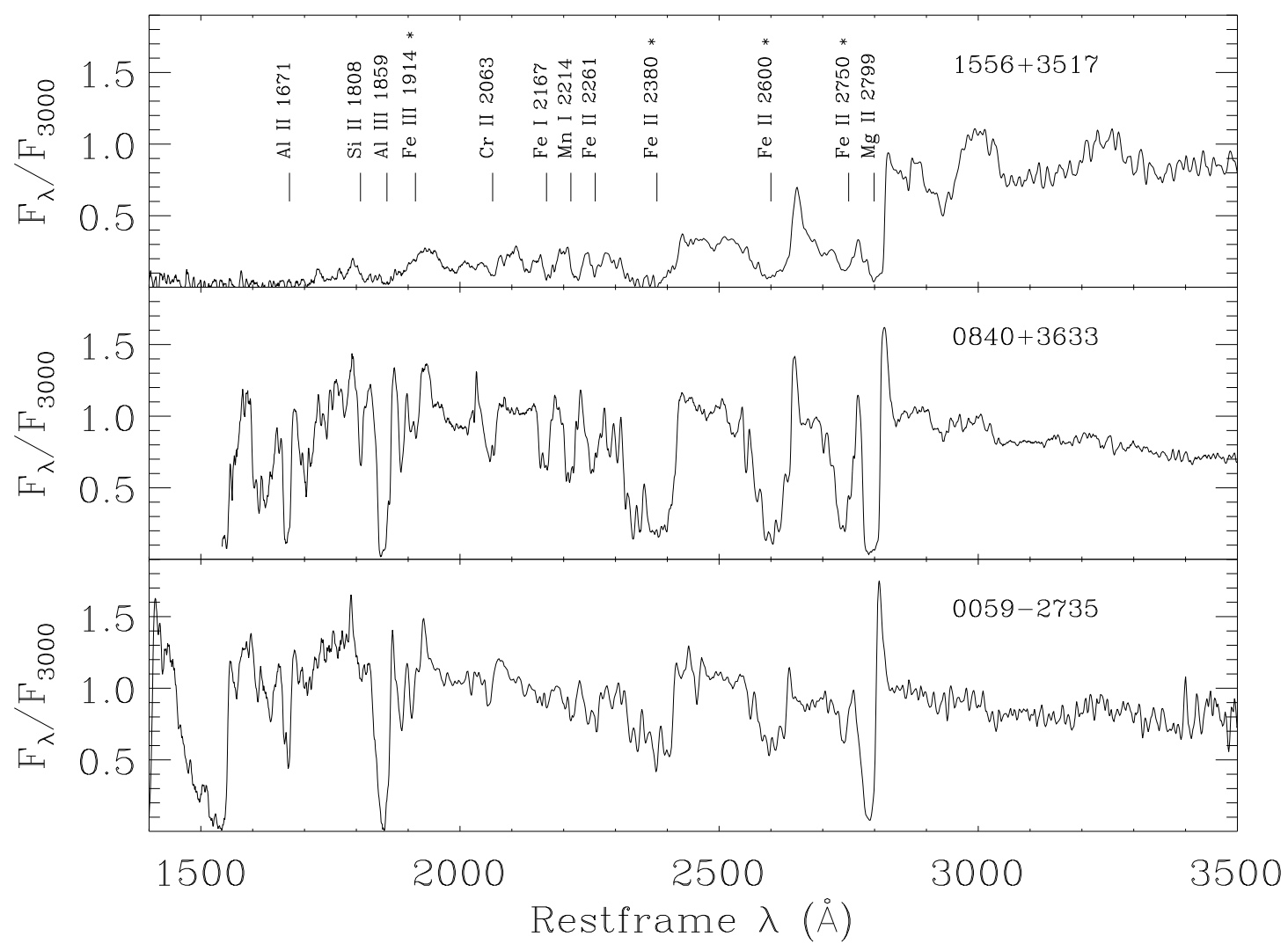

Fig. 1. - Lick Kast 3m spectra of $0840+3633$ and $1556+3517$ compared to the spectrum of 0059-2715 from Weymann et al. (1991). Prominent absorption features are marked; an asterisk denotes metastable states of iron. 